\title{
COMENTARIO
}

\section{Las deudas de los oprimidos en el imperio de la liquidez}

\author{
Jean-Michel Servet
}

$\mathrm{L}$ os trabajos que anteceden a este texto tienen en común un punto original: captan los fenómenos financieros a través de las prácticas de las comunidades y sus miembros y lo hacen básicamente a microescala, lo cual implica integrar la experiencia de los actores. Por lo general, las finanzas se estudian en una perspectiva global, se establece el vínculo entre la tasa de inclusión financiera econométricamente y sobre todo el nivel del ingreso per capita o mediante las modalidades legales y reglamentarias que enmarcan y legitiman lo que se interpreta como una confrontación de ofertas y demandas. Así, queda oculta la dimensión de las finanzas como una relación social determinante. Ahora bien, las prácticas llamadas "financieras" tienen por objeto social, político, económico, cultural, etcétera -según el contenido específico que las culturas y las ideologías dan a estas categorías-, vincular las comunidades y a sus miembros. Lo hacen en el tiempo y en el espacio, a través de la deuda y el crédito que constituyen e instituyen las finanzas. Implican obligaciones, recíprocas o no, y expresan relaciones de solidaridad y de dominio entre financieros y financiados. Esta relación puede ser también de empleo, como lo muestra el ejemplo de la servidumbre por deudas - bonded labour - . Vemos que esto ocurre mediante el depósito de una fianza o garantía personal o solidaria. El sustrato de la relación financiera es monetario en el sentido de que se realiza con medios de pago y unidades de cuenta - tanto los unos como las otras pueden adoptar distintas formas-. Lo monetario se materializa también a través de cualquier tipo de reserva ${ }^{1}$ que garantice la capacidad de existencia de una colectividad o el dominio de quien o quienes la controlan sobre quienes son dependientes. ${ }^{2}$ Comprender de esta manera las prácticas financieras, es decir, como un vínculo, es otra forma de expresar que funcionan como una relación social que, aun cuando parezca interindividual, no

\footnotetext{
${ }^{1}$ Esta definición difiere de la visión acostumbrada que toma en cuenta la función de reserva de la moneda como preservación en el tiempo del poder adquisitivo, lo cual hace que tal enfoque no le dé en realidad ninguna autonomía de la llamada función de pago ni de la de cuenta.

${ }^{2}$ Desarrollo esta definición no funcional de lo monetario inspirándome en gran medida en las propuestas de Polanyi (Servet, 2012).
}

The Debts of the Oppressed in the Empire of Liquidity

Jean-Michel Servet: Institut des Hautes Études Internationales et du Développement, Ginebra, Suiza jean-michel.servet@graduateinstitute.ch

Desacatos, núm. 44, enero-abril 2014, pp. 103-109 
puede existir más que en dimensiones colectivas esenciales. Así, las redes que se tejen no pueden limitarse a una confrontación mecánica entre intereses a la cual podría reducirse la lógica de los mercados. Hay un reconocimiento del doble nivel en esta dinámica: interindividual y colectivo. Estas relaciones aparecen como un ingrediente fundamental de la interdependencia de las actividades y los actores, para bien y, con frecuencia aquí, para mal...

$\mathrm{Y}$ es que otro elemento notable en los análisis y testimonios acerca de situaciones y países muy distintos es el alcance no sólo del endeudamiento, sino en especial del sobreendeudamiento. Todos plantean de una u otra forma la cuestión misma de su definición, ya que el sobreendeudamiento no puede entenderse simplemente como la incapacidad absoluta en que se hallaría una persona, una familia o un grupo de "garantía solidaria" para enfrentar los vencimientos de sus deudas. El problema esencial no es en primer término la imposibilidad de pagar, sino lo contrario: que el deudor pague todo o en parte empobreciéndose más y más. Los trabajos muestran las mil y una estratagemas de que echan mano las poblaciones para lograrlo y sobrevivir. Se ve a partir de ejemplos, sobre todo indios, cuáles son las jerarquías sociales y morales de las deudas. No obstante, lo que la observación de los vínculos de deuda no revela, o lo hace de manera parcial, es el sistema económico y financiero global que determina el conjunto y actúa de manera dominante. El trabajo de Magdalena Villarreal se refiere a esto al examinar el tema de los préstamos inmobiliarios contratados por los migrantes mexicanos en los Estados Unidos, una de las bases más conocidas de la crisis de las hipotecas de alto riesgo - subprimes-. Profundicemos en su análisis.

Básicamente, la crisis ${ }^{3}$ se deriva de políticas de corte neoliberal - privatización, desregulación,

\footnotetext{
${ }^{3}$ Para una discusión más amplia, véase Servet (2010b). Acerca del sobreendeudamiento, véase el artículo complementario de Saiag y Servet (en prensa). Estos trabajos coinciden con las conclusiones de los análisis macroeconómicos de Wade (2011).
}

etc. - basadas en la creencia no sólo en la capacidad de los mercados de autorregularse - a lo cual podría responder un control de los mercados-, sino también en una distribución cada vez más desigual tanto de los ingresos como de los patrimonios, en los ámbitos nacional y mundial. La proporción de los salarios en la distribución del ingreso ha disminuido desde principios de la década de 1980 y ha sido causa de una insuficiencia de la demanda global durante un periodo limitado por el aumento del endeudamiento de las unidades domésticas. Se registra también un crecimiento correlativo de los ingresos financieros en comparación con otras fuentes de ingresos, sobre todo en relación con la gestión de los riesgos, lo cual ha derivado en una inestabilidad de las economías cada vez mayor. Los textos demuestran ampliamente el empobrecimiento relativo de las poblaciones, incluso en términos subjetivos, debido a una explosión de las necesidades provocada en particular por una mundialización creciente de las economías y por una ideología según la cual poseer vale más que ser. Tales son las causas profundas de la crisis, pues la desigualdad cada vez mayor de los patrimonios y los ingresos ha desembocado en una desaceleración de la demanda global y en una acumulación financiera que esteriliza e incluso estrangula cada vez más la economía real. Dado el dominio sobre ésta de los mercados financieros, de los movimientos especulativos y del enriquecimiento de quienes directa o indirectamente se benefician de ellos, hemos llegado a un punto en que los precios de mercado están muy lejos de contribuir de forma efectiva a la creación de riqueza. Puede hablarse en este sentido de un imperio de la liquidez. La creciente facilidad y la desregulación de las transacciones y los intermediarios son resultado de la "financiarización" de los intercambios mercantiles. Con frecuencia se pone de relieve que hay un proceso de mercantilización generalizada, sin que se tome en cuenta que el funcionamiento de las economías y la moral misma han desplazado las fronteras de lo que se puede comprar y vender - así, el comercio legal de esclavos fue desapareciendo 


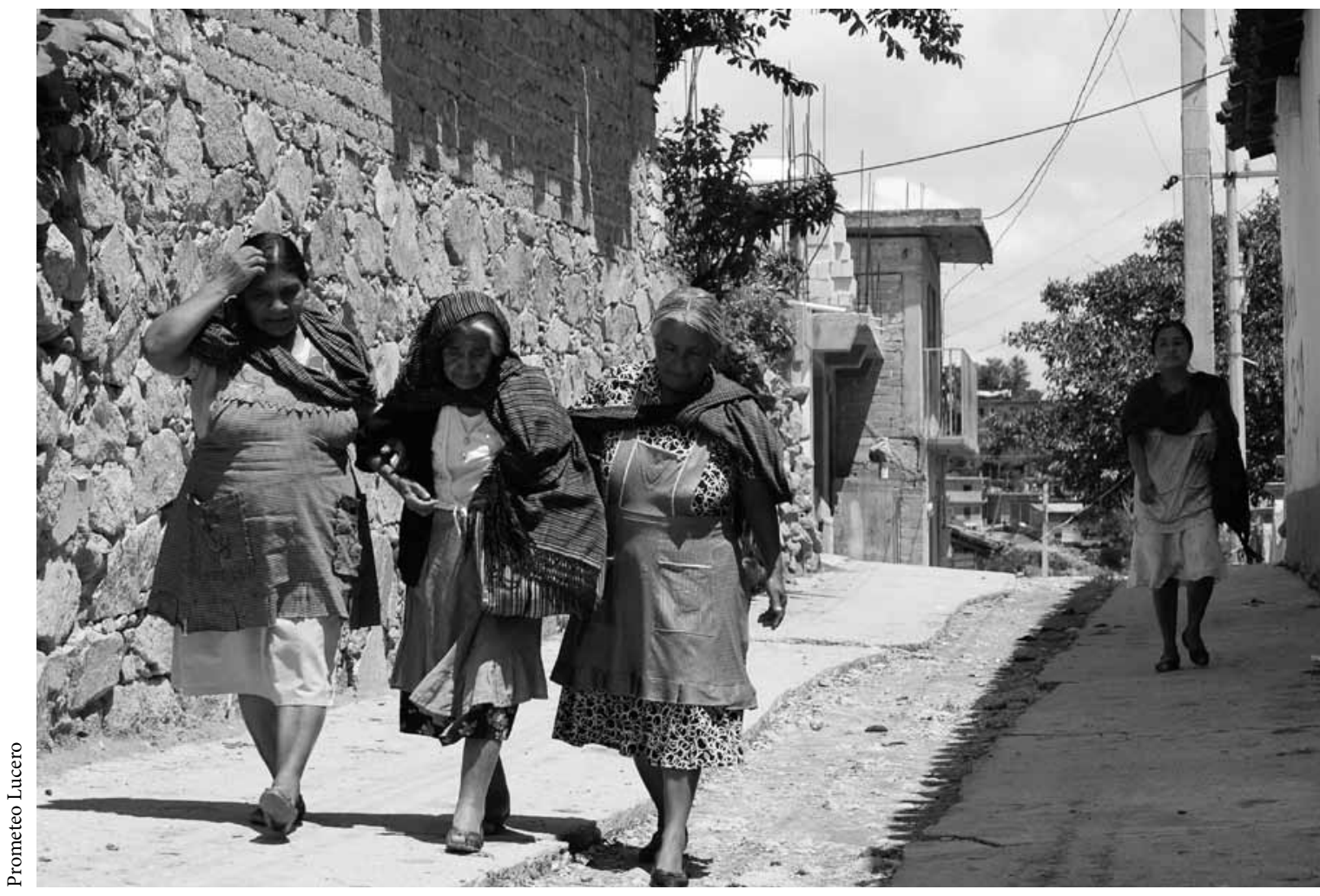

Mujeres nahuas acuden a la comisaría de Chiepetepec para recibir el apoyo de Oportunidades.

progresivamente en el siglo xIX, lo cual constituyó en sí una reducción de la mercantilización-. Los cambios esenciales se ven determinados por la "financiarización” generalizada. Un subproducto de ésta son las formas contemporáneas de servidumbre por deudas, al igual que los famosos préstamos inmobiliarios subprime en Estados Unidos, donde el valor de la deuda se desplomó debido a una recesión del mercado inmobiliario. Se trata de diferentes facetas de un mismo conjunto en todo el mundo. No es una suma de problemas, de crisis, sino una crisis global de los modos económicos de producción, de intercambios y de consumo, cuyo elemento vinculador son las finanzas.

Pero el endeudamiento e incluso la especulación sobre las deudas no son fenómenos nuevos. No obstante, hay una gran diferencia entre los movimientos especulativos de antes y los que ha padecido y padece todavía casi la totalidad del planeta. Se advierte un cambio esencial respecto de las antiguas formas de especulación: si antes para cada operación se podía decir quién cedía y quién adquiría, o quién era intermediario, actualmente los mismos agentes $\mathrm{u}$ organizaciones ocupan indistintamente y por turnos la posición de vendedores o de compradores de los mismos títulos de valores o acciones, según sus expectativas y previsiones de mercado. Se invalida cada vez más el argumento de financiar una producción o su transporte y por tanto de retener o deducir un margen que, en ciertos casos, puede rayar en la explotación, y la justificación que se da al papel de los intermediarios en esos mercados entre los productores y los destinatarios finales ha perdido su base de legitimidad. La velocidad de circulación de los títulos o acciones que representan las deudas y las producciones a futuro ilustra la desconexión que 
favorecen respecto de los movimientos reales de los bienes y los servicios. Hoy en día, en la cima de la pirámide financiera, los actores de esos movimientos especulativos son sobre todo los fondos de pensiones y los hedge funds, que movilizan billones de dólares. De ahí surge un principio de liquidez: todas las mercancías pueden convertirse en cierta forma en dinero, más allá de sus características de uso y sin guardar proporción con las necesidades productivas y de consumo final. La liquidez de esos mercados, dicho de otra manera, la disponibilidad de los títulos y acciones y la capacidad de venderlos, permite que ocurran muchísimas idas y vueltas en un mismo día, como sucede con las divisas, otra joya de la corona de esta economía especulativa. El título que representa una tonelada de trigo es objeto, en promedio, de 2000 transacciones entre su creación a partir de la producción potencial cedida y la entrega de la mercancía. Estos movimientos especulativos hacen posible atraer sumas considerables mediante las finanzas y los servicios conexos - principal- excluidas. Se requiere de una serie de transformaciones de los títulos financieros para permitir que haya una pirámide de especulaciones y de acumulaciones expoliadoras. La base de la creación de riqueza se ve desplazada de una lógica real que articula la producción y el consumo a una de apropiación de recursos, principalmente gracias a operaciones que se presentan como puramente financieras y que muestran todos los estigmas de la modernidad. No obstante, la desproporción entre la producción a base de trabajo y estas finanzas depredadoras había llegado a tal grado que el desequilibrio de ese sistema no podía sino ser cada vez mayor, de ahí la crisis que se desencadenó en 2007-2008.

Hay quienes afirman, no sin razón, que resulta inútil denunciar los vicios de esta economía financiarizada y sus consecuencias para las poblaciones más vulnerables. Pero hay que saber proponer soluciones o alternativas que no pueden limitarse a buenos deseos ni a consejos morales. ¿Qué proponer? En este contexto, hay voces que claman por regresar a un poder público, en los ámbitos nacionales e internacional, que controle las transacciones financieras y las haga pagar impuestos. Alegan que tales impuestos permitirían también, por ejemplo, fortalecer la ayuda a los países en desarrollo y compensar los déficit públicos. Sin embargo, lo que fue un buen tema de debate para revelar las tensiones mortales entre finanzas, economía y sociedad no puede constituir una respuesta eficaz a la crisis, ya que no produce ningún cambio fundamental en las articulaciones entre finanzas, producción y consumo, o más en general en la organización financiera y económica de las sociedades. Modifica de manera marginal la distribución de los ingresos, limita las retenciones financieras para dar el poco oxígeno necesario para... que no haya cambios fundamentales en la organización económica y financiera de las sociedades y, en consecuencia, en el reparto de los ingresos y los patrimonios. Sin duda conviene gravar con impuestos esas ganancias, como todas las otras, no necesariamente las transacciones en sí, ya que si se toma el ejemplo del mercado inmobiliario, 
un sector particularmente sometido a gravámenes, es de hacer notar que éstos no impiden la especulación. Así pues, gravar las transacciones financieras no eliminaría las finanzas especulativas y expoliadoras, lo que es peor: las legitimaría. Si consideramos nocivas y hasta inmorales esas especulaciones, habría que prohibirlas. No se le ocurriría a nadie legalizar el exceso de velocidad, el envenenamiento de alimentos o las desviaciones sexuales para cobrar impuestos con el fin de financiar las obras de asistencia para... las víctimas de esos automovilistas, para desintoxicar a los envenenados o abrir albergues para los niños víctimas de abusos sexuales. Los impuestos que podrían castigar las finanzas han sido comparados, erróneamente, con las célebres indulgencias a las que se opuso Lutero. Pero la diferencia de la lógica de cada uno de esos casos es inmensa. Quien paga impuestos queda en cierta forma justificado, mientras que las indulgencias no salvan del infierno: quien las obtiene sigue siendo pecador, sólo tiene la posibilidad de reducir su estancia en el purgatorio. Los especuladores, al igual que los contaminadores del ambiente, consideran que el sistema impositivo les permite redimirse en términos morales y continuar sus actividades sin apenas compensar los efectos dañinos de sus intervenciones.

¿Cuáles pueden ser las respuestas de las finanzas y de la economía solidaria a este cada vez mayor imperio de la liquidez? Veamos algunos ejemplos de lo que se puede reunir bajo la bandera de la solidaridad, ciertos testimonios de las articulaciones posibles tanto con el mercado como con el Estado - pues las prácticas financieras solidarias no se oponen ni a uno ni al otro-- Suelen ser más conocidas las iniciativas de economía solidaria que las de finanzas solidarias. El comercio equitativo es sin duda una de las formas más populares, así como los intercambios basados en circuitos cortos de distribución para acercar a los consumidores con los productores. Estas prácticas solidarias también tienen una dimensión financiera, ya que garantizan la regularidad del flujo de financiación a los productores. Las finanzas solidarias propiamente dichas abarcan un amplio espectro de iniciativas y de innovaciones. Citemos en primer lugar las monedas complementarias o alternativas que pueden contribuir al desarrollo local en determinado territorio. Esas monedas suponen la existencia de grupos, cada uno de los cuales constituye una organización que admite, de modo más o menos formalizado, relaciones de débito y de crédito entre sus miembros o que emite la moneda con la que se harán los pagos. La capacidad de autonomía monetaria de cada grupo le permite crear su moneda ex nihilo, a raíz del otorgamiento a sus miembros del primer adelanto o mediante la adquisición de esas monedas locales con moneda nacional. Las monedas complementarias manifiestan un potencial de apoyo al desarrollo localizado que es susceptible de ser superior al del microcrédito, por ejemplo, debido a un mecanismo de endogenización de los recursos, es decir, que alguien que tiene moneda local sólo puede dirigirse con ese medio de pago a un productor local de bienes o servicios. Así, sólo hay una fuga limitada de recursos fuera de la economía local. ${ }^{4}$ Tras reaparecer en Canadá en la década de 1980 y en Estados Unidos en la de 1990, esas monedas locales luego fueron surgiendo en cada vez más países -en Argentina es donde están más extendidas- $-{ }^{5} \mathrm{Su}$ reconocimiento institucional gana terreno sobre todo entre colectividades territoriales.

Pese a sus numerosas derivas, aún es posible mencionar el microcrédito, sin omitir que hay que cuidarse de no tirar al niño con el agua de la bañera. Porque una de las características del microcrédito es que las instituciones que lo difunden no son objeto de cotización en bolsa y de facto los títulos, ya sea préstamos o tomas de participación, son en general difícilmente transferibles y en consecuencia tienen una liquidez

\footnotetext{
${ }^{4}$ Desarrollo el argumento en Servet (2010a). Acerca de las monedas complementarias en el mundo, véase la vasta producción de Jérôme Blanc (Université Lyon 2) sobre el tema.

${ }^{5}$ Entre los numerosos trabajos sobre el tema, véase la tesis de Saiag (2011), que distingue dentro del movimiento de monedas locales en Argentina algunas que obedecen en gran medida al principio de solidaridad y otras que sólo son un sustituto del mercado.
} 


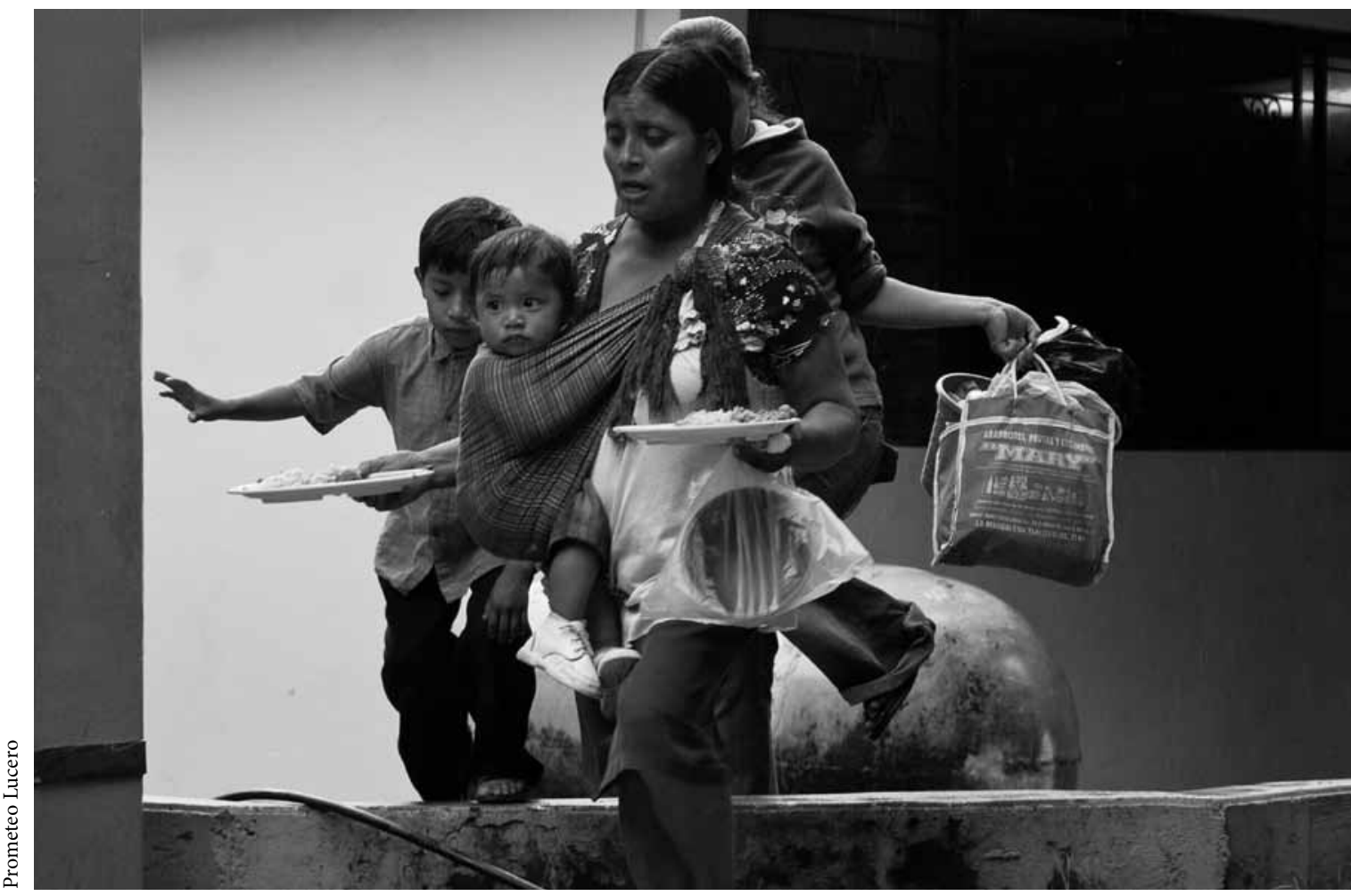

Familias enteras parten de Tlapa, abordan autobuses desde la Unidad de Servicios Integrales para trasladarse a campos de cultivo en el norte, septiembre de 2010 .

muy débil. Por tanto, no son susceptibles de ser objeto de especulaciones a plazos. Hoy en día, las microfinanzas en sus formas más comunes se oponen así al imperio de la liquidez. Entre las iniciativas actuales que buscan combinar el microcrédito con el principio de las monedas locales, el ejemplo más conocido es el del Banco Palmas, en Fortaleza, y sus similares en unas 50 localidades brasileñas. En 2002, Social Trade Organisation (sTro), una organización holandesa, y actualmente el Banco Nacional de Brasil, han depositado fondos en esta institución de microcrédito. Para evitar hacer préstamos en moneda nacional que permitirían que quienes los reciben compraran productos externos, el banco que emite la moneda local conserva la cantidad correspondiente en un banco bajo la forma de depósito y crea el equivalente en moneda local. Esa moneda local que luego se presta permite impulsar una dinámica de desarrollo localizado. La dificultad está en el hecho de que si quienes reciben en pago la moneda local la llevan de inmediato al banco local para convertirla en moneda nacional, y no la usan como poder de compra de bienes y servicios producidos en su localidad, prácticamente se anulará el impacto interno de dinamización de los intercambios locales y en consecuencia también los efectos multiplicadores sobre la producción de bienes y servicios. Otro ejemplo de finanzas solidarias opuestas en los hechos al imperio de la liquidez es el Fondo Internacional de Garantía (FIG), cuya sede central fue fundada en Ginebra por el movimiento mutualista de tipo Raiffeisen, que permite movilizar fondos en los bancos de países latinoamericanos o del oeste de África para que sean prestados localmente. Es una de las primeras organizaciones de este tipo y existen unas 60 en todo el mundo. Una vez más se trata de 
financiar de otra manera que no sea mediante inversiones especulativas.

Sin duda, por originales que sean, esas iniciativas no son la gran panacea. Pueden parecer muy humildes ante la magnitud y la diversidad de formas de la opresión económica y financiera en todos los continentes de la que son testigos los análisis que se presentan en este número de Desacatos. Resulta evidente que no se puede luchar por eliminar en forma rápida y eficaz las múltiples formas de esclavitud por deudas sólo con pequeños créditos para iniciar nuevas actividades generadoras de ingresos ni mediante el efecto multiplicador de las microinversiones sobre los ingresos. Es indispensable contar con regulaciones para promover el empleo decente para todos $y$, correlativamente, con normas para informar a los compradores sobre las calidades, incluyendo las sociales, de los productos puestos a la venta y, en ciertos casos, para prohibir ciertas producciones a bajo costo. Es ilusorio creer que las medidas financieras pueden por sí solas permitir que nos protejamos de manera individual y colectiva contra los sucesivos embates del imperio de la liquidez. Por humildes que parezcan estas iniciativas, y se trata de una característica esencial que distingue la economía solidaria de la economía mercantil, son materia de debate y por ello contribuyen a la vida democrática al vincular lo económico y lo financiero con lo político. La democracia puede ser la principal arma de los más débiles para liberarse incluso de los obstáculos económicos. En términos generales, las prácticas de la economía y las finanzas solidarias están expuestas a permanente arbitraje, ya que hacen que todos sean actores de sus gastos, de sus eventuales inversiones, de su ahorro, de sus préstamos, etcétera, por humildes que sean. Las interrogantes no implican respuestas siempre sencillas, pues los presupuestos se ven tanto más constreñidos en cuanto que los recursos son escasos y, sobre todo, que no pueden ser, más que en casos excepcionales, de la índole del todo o nada.

\section{BIBLIOGRAFÍA}

Saiag, Hadrien, 2011, “Le trueque argentin au prisme de la dette: une socioéconomie des pratiques monétaires et financières", tesis de doctorado, Universidad ParísDauphine, París.

- y Jean-Michel Servet, en preparación, "Household Over-indebtedness in Contemporary Societies: A Macro-perspective", en Isabelle Guérin, Solène Morvant-Roux y Magdalena Villarreal (eds.), Over-Indebtedness and Financial Inclusion.

Servet, Jean-Michel, 2010a, "Microcredit", en Keith Hart, Jean-Louis Laville y Antonio David Cattani (eds.), The Human Economy, Polity Press, Boston, Cambridge, Oxford, pp. 130-141.

- 2010b, Le grand renversement: de la crise au renouveau solidaire, Desclée de Brouwer, París.

, 2012, Les monnaies du lien, Presses Universitaires de Lyon, Lyon.

Wade, Robert H., 2011, "Income Inequality: Should we Worry about Global Trends?", en European Journal of Development Research, núm. 23, pp. 513-520. 